\begin{tabular}{ccc}
\hline & International Journal of Engineering \& Technology, $7(3.29)(2018) 170-172$ \\
SPC & Website: www.sciencepubco.com/index.php/IJET \\
Research paper & Jechnology \\
\hline
\end{tabular}

\title{
Theory of a superconducting artificial neuron for extended backpropagation learning algorism
}

\author{
Haruna Katayama ${ }^{1}$ *, Toshiyuki Fujii ${ }^{2}$, Noriyuki Hatakenaka ${ }^{3}$ \\ ${ }^{1}$ Faculty of Integrated Arts and Sciences, Hiroshima University, 1-7-1 Kagamiyama, Higashi-Hiroshima, 739-8720, Japan \\ ${ }^{2}$ Department of Physics, Asahikawa Medical University, 2-1-1-1 Midorigaoka-higashi, Asahikawa, 078-8510, Japan \\ ${ }^{3}$ Graduate School of Integrated Arts and Sciences, Hiroshima University, 1-7-1 Kagamiyama, Higashi-Hiroshima, 739-8720, Japan \\ *Corresponding author E-mail: noriyuki@ hiroshima-u.ac.jp
}

\begin{abstract}
An artificial neuron using superconducting devices, so-called double SQUID, applicable to the extended backpropagation learning algorism is studied. It is shown that the tunable slope of the sigmoid function required in the algorism can be achieved under the fixed temperature by externally applied magnetic fields threading the ring with two Josephson junctions in the double SQUID.
\end{abstract}

Keywords: Artificial Neural Networks; Superconducting Quantum Interference Devices (SQUID); Sigmoid Function; Superconducting Neurons.

\section{Introduction}

There has been considerable interest in artificial intelligence capable of dealing with intractable tasks in conventional manner under an uncertain environment. An artificial neural network (ANN) inspired by biological nervous networks is a key technology to support artificial intelligence [1].

(A)

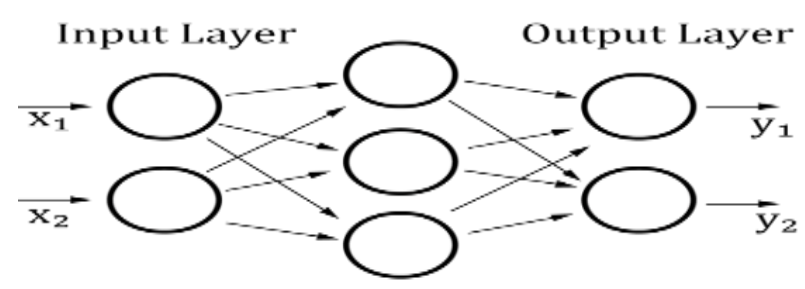

Hidden Layer

(B)

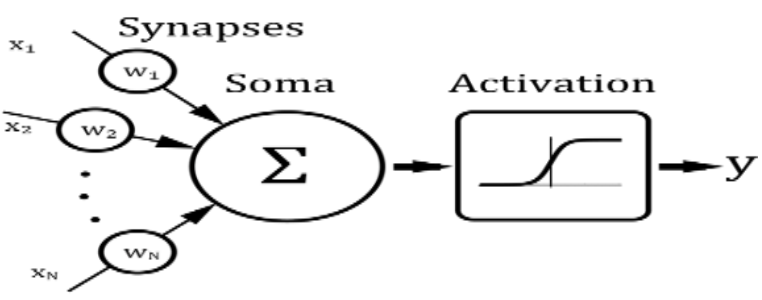

Fig. 1: Schematic Diagrams of (A) A Feed-Forward Artificial Neural Network and (B) an Artificial Neuron.

ANN is a model of actual neural networks and comprises many neuron elements arranged in the layered structure, which are interconnected to one another via synapse elements as shown in Fig. 1(a). Each neuron receives input signals from the neurons of the previous layer and outputs the signal to the neurons of the subsequent layer according to the activation function at Soma (see Fig. 1(b)). Thus, neurons do not output all received input signals. The activation function that produces a nonlinear response representing the threshold is the center of the neuron. Among the nonlinear response functions as the activation or transfer function in neurons, sigmoid functions are significantly effective in making ANN implementing the backpropagation (BP) learning algorism [2] for simple mathematical handling, especially their differentiation. In the extended BP learning algorism aiming at higher learning efficiency [3], the slope of the sigmoid function in each neuron is also a learning target in addition to the weighted coefficients in ANN. Therefore, artificial neurons described in the sigmoid function with a tunable slope (or gradient) are required.

In this paper, we analyze a superconducting quantum interference devices (SQUIDs) with tunable Josephson tunnel current, so-called double SQUID, as a candidate for an artificial neuron in ANN implemented tunable slope of the sigmoid function for enhancing learning efficiency in the extended BP learning algorism.

SQUID-based artificial neurons

Superconducting devices are a promising candidate for realizing ANN because of their ultra-high speed operation, ultra-low power consumption, and scalability enabled by nanotechnology. In fact, ANN using superconducting circuits has already been implemented in various ways [4]-[10]. Here, we propose a superconducting artificial neuron applicable to the extended BP learning algorism which requires variable slope of the sigmoid function.

a) Physical origin of sigmoid function generation

In our previous paper [11], we clarified the physical origin of the sigmoid function generation. i.e., the sigmoid function is generated in the transition processes between two states in double well potential. The probability of finding a particle in one of the two wells, $p$, in thermal equilibrium is derived from the rate equation on the transition between two states in the double well potential as follows;

$p=\frac{1}{1+\mathrm{e}^{-\Delta \mathrm{E} / \mathrm{kT}}}$

Where $\Delta \mathrm{E}$ is the energy difference between two states? $\mathrm{k}$ and $\mathrm{T}$ are the Boltzmann constant and temperature, respectively. It can be 
seen that the slop of the sigmoid function can be changed by controlling the temperature. However, it is technically difficult simply to control temperature of neurons. The individual control increases its difficulty. Therefore, the temperature control of a neuron is not realistic, and then a simpler control method is required.

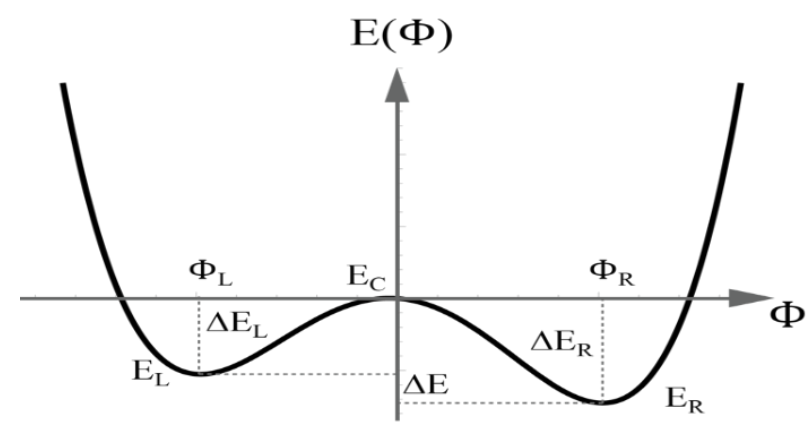

Fig. 2: Schematic Diagram of Double Well Potential.

b) SQUID-based artificial neurons

Based on the two-state transition scheme for generating the sigmoid function to artificial neurons, we analyzed radio frequency (rf) SQUID as a superconducting artificial neuron, which consists of a superconducting loop with the inductance L interrupted by a Josephson junction (JJ) as shown in the middle of Fig. 2 (a).

The Hamiltonian of rf-SQUID is given by

$\mathrm{H}=\mathrm{E}_{\mathrm{L}}\left(\widehat{\Phi}-\widehat{\Phi}_{\mathrm{ex}}\right)^{2}+\mathrm{E}_{\mathrm{J}}\{1-\cos (2 \pi \widehat{\Phi})\}$

where $\widehat{\Phi}$ and $\widehat{\Phi}_{\text {ex }}$ are magnetic flux through the superconducting ring and an externally applied magnetic flux normalized by the quantum unit of magnetic flux $\widehat{\Phi}_{0}=\mathrm{h} / 2 \mathrm{e}$ with $\mathrm{h}$ and e being Planck's constant and an elementary electric charge, respectively. The first term represents magnetic energy accumulated in the ring with $\mathrm{E}_{\mathrm{L}}=\widehat{\Phi}_{0}^{2} / 2 \mathrm{~L}$. The second term represents Josephson coupling energy given by $\mathrm{E}_{\mathrm{J}}=\hbar \mathrm{I}_{\mathrm{C} 0} / 2 \mathrm{e}$ with $\mathrm{I}_{\mathrm{C} 0}$ being the Josephson critical current. Fig. 2 (b) shows the potential profile as a function of $\widehat{\Phi}$ with different applied magnetic flux values $\widehat{\Phi}_{\mathrm{ex}}$. The lowest two minima form a double well potential required to generate the sigmoid function.

The energy difference $\Delta \mathrm{E}$ in (1) is given as

$\Delta \mathrm{E}\left(\widehat{\Phi}_{\mathrm{ex}}\right)=\mathrm{E}\left(\widehat{\Phi}_{\mathrm{L}}\right)-\mathrm{E}\left(\widehat{\Phi}_{\mathrm{R}}\right)=\mathrm{E}_{\mathrm{L}}\left[\left(\widehat{\Phi}_{\mathrm{R}}+\widehat{\Phi}_{\mathrm{L}}\right)-\right.$

$\left.2 \widehat{\Phi}_{\mathrm{ex}}\right]+2 \mathrm{E}_{\mathrm{J}} \sin \left(\pi\left(\widehat{\Phi}_{\mathrm{R}}+\widehat{\Phi}_{\mathrm{L}}\right)\right) \sin \left(\pi\left(\widehat{\Phi}_{\mathrm{R}}-\widehat{\Phi}_{\mathrm{L}}\right)\right)$

where $\widehat{\Phi}_{\mathrm{L}(\mathrm{R})}$ is the magnetic flux giving the local minimum on the left (right) side of the double well potential and is given as a solution to the following equation

$$
\sin (\pi \widehat{\Phi})=-\frac{1}{\pi \alpha}\left(\widehat{\Phi}-\widehat{\Phi}_{\mathrm{ex}}\right)
$$

Where $\alpha=E_{J} / E_{L}$. By using an approximate solution for analytically unsolved $\widehat{\Phi}_{\mathrm{L}(\mathrm{R})}$, we obtained $\Delta \mathrm{E} / \mathrm{E}_{\mathrm{L}}$ in the linear form of $\widehat{\Phi}_{\mathrm{ex}}$ as

$\Delta \mathrm{E} / \mathrm{E}_{\mathrm{L}}=\frac{4 \pi^{2} \alpha}{1+2 \pi^{2} \alpha}\left[2\left(\widehat{\Phi}_{\mathrm{R} 0}-\widehat{\Phi}_{\mathrm{L} 0}\right)-1\right] \widehat{\Phi}_{\mathrm{ex}}+$ const.

Where $\widehat{\Phi}_{\mathrm{L} 0(\mathrm{R} 0)}$ is the magnetic flux at an unbiased case? It turns out that the slope depends only on $\alpha$ since the value in the braces of the first term is almost unity [11]. In other words, we can change the slope by controlling the parameter $\alpha$.
(A)

double SQUID

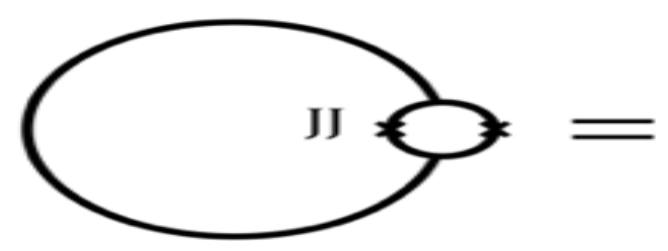

(B)

$\mathrm{E}\left(\Phi / \Phi_{\mathrm{O}}\right)$

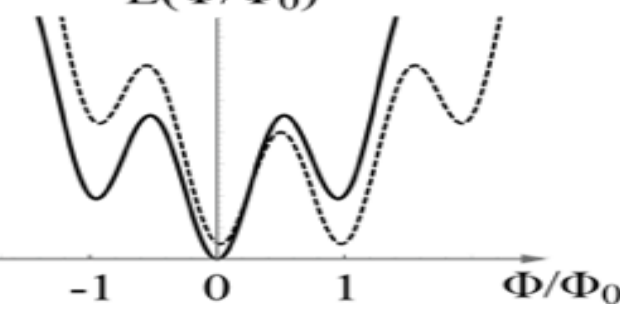

(C)

rf SQUID

dc SQUID

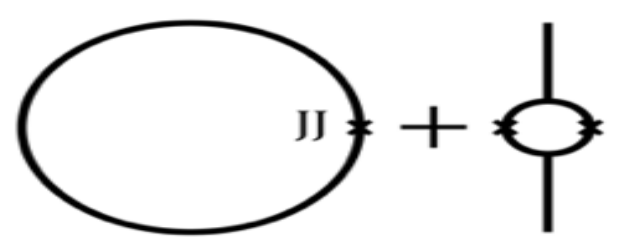

(D)

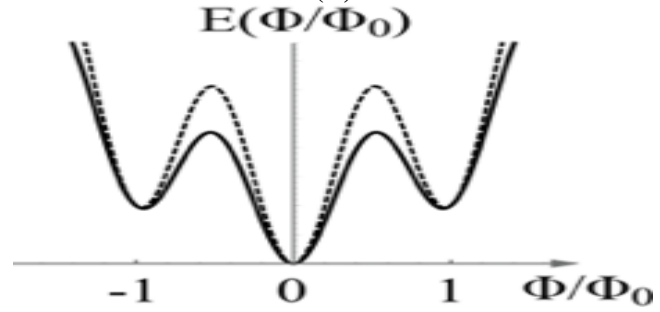

Fig. 3: Schematic Diagram of (A) Double SQUID Comprising of RfSQUID with Dc-SQUID and its Potential Profile as A Function of Magnetic Flux Threading Both (B) the Rf-SQUID Ring With $\widehat{\Phi}_{\mathrm{Ex}}=0$ (Solid Line) And $\widehat{\Phi}_{\mathrm{Ex}}=0.5$ (Dotted Line) and (C) the Dc-SQUID Ring With $\widehat{\Phi}_{\mathrm{C}}=0$ (Solid Line) and $\widehat{\Phi}_{\mathrm{C}}=0.4$ (Dotted Line).

Sigmoid function with tunable slope

Now let us consider a superconducting artificial neuron described by the sigmoid function with a tunable slope. In order to introduce a variable slope into rf-SQUID, it is sufficient to control the parameter $\alpha$, i.e., the Josephson coupling energy $E_{J}$ under the built-in inductance.

It is well known that the variable or tunable Josephson coupling energy can be achieved by direct current (dc) SQUID, which is also a SQUID consisting of a superconducting loop with two Josephson junctions as shown in the right side of Fig. 2 (a). The current through the dc-SQUID can be described by

$$
\mathrm{I}_{\mathrm{c}}\left(\widehat{\Phi}_{\mathrm{c}}\right)=2 \mathrm{I}_{\mathrm{C} 0} \cos \left(\pi \widehat{\Phi}_{\mathrm{c}}\right)
$$

Where $\widehat{\Phi}_{\mathrm{c}}$ is a magnetic flux threading the loop in dc SQUID normalized by $\widehat{\Phi}_{0}$. Thus, a double SQUID, which is a combined superconducting device replacing the Josephson junction in rf-SQUID with dc-SQUID, behaves as normal rf-SQUID with tunable Josephson critical current, resulting in a tunable Josephson coupling energy $\mathrm{E}_{\mathrm{J}}\left(\widehat{\Phi}_{\mathrm{c}}\right)=\hbar \mathrm{I}_{\mathrm{c}}\left(\widehat{\Phi}_{\mathrm{c}}\right) / 2$ e. Fig. 3 shows sigmoid functions with different magnetic flux $\widehat{\Phi}_{c}$ threading the loop in dc SQUID. This clearly demonstrates that the slope of the sigmoid function is controlled by changing the applied magnetic flux without changing temperature. Therefore, double SQUID acts as an artificial neuron 
for the extended BP learning algorism in stochastic artificial neural networks.

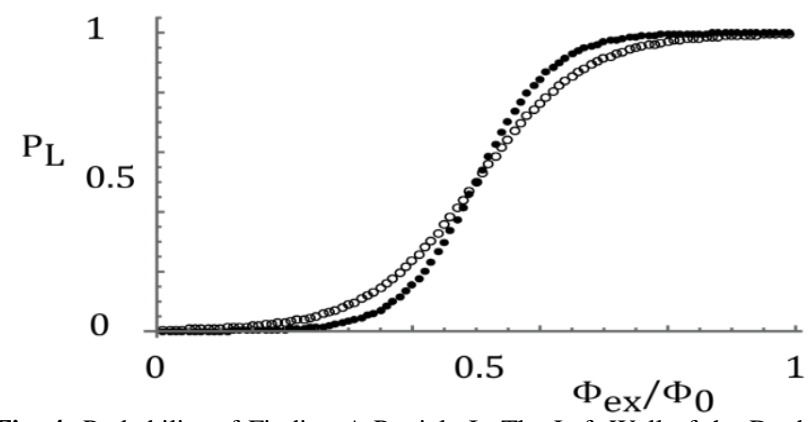

Fig. 4: Probability of Finding A Particle In The Left Well of the Double Well Potential as A Function of Magnetic Flux $\widehat{\Phi}_{e x}$ in Rf SQUID With Different Magnetic Flux $\widehat{\Phi}_{c}$ in Dc SQUID At The Fixed Temperature $E_{L} / k T=$ $10 \operatorname{And} \alpha=1$. The Filled and Open Circles Are $\widehat{\Phi}_{c}=0$ and $\widehat{\Phi}_{c}=0.4$, Respectively.

\section{Conclusion}

We have presented an artificial neuron using double SQUID applicable to the extended BP learning algorism developed to enhance leaning efficiency. The variable slope of the sigmoid function required for the extended BP learning algorism can be achieved by an externally applied magnetic flux at constant temperature in the double SQUID. Therefore, the proposed double SQUID-based superconducting artificial neuron, which can individually control neurons by the existing technology, has superior controllability compared with conventional artificial neurons, which can be controlled only by temperature changes.

\section{References}

[1] F. Rosenblatt, Principles of Neurodynamics: Perceptrons and the Theory of Brain Mechanisms (Spartan Books, Washington, 1962).

[2] D. E. Rumelhart, G. E. Hinton, and R. J. Williams, "Learning representations by back-propagating errors," Nature, vol. 323, pp. 533536, October 1986.

[3] A. Sperduti and A. Starita, "Speed up learning and network optimization with extended back propagation," Neural Networks, vol. 6, pp. 365-383, 1993.

[4] Y. Harada and E. Goto, "Artificial neural network circuits with Josephson devices," IEEE Transactions on Magnetics, vol. 27, pp. 2863-2866, March 1991.

[5] M. Hidaka and L. A. Akers, "An artificial neural cell implemented with superconducting circuits," Superconductor Science and Technology, vol. 4, pp. 654-657, November 1991.

[6] Y. Mizugaki, K. Nakajima, Y. Sawada, and T. Yamashita, "Superconducting neural circuits using fluxon pulses," Applied Physics Letters, vol. 62, pp. 762-764, February 1993.

[7] Y. Mizugaki, K. Nakajima, Y. Sawada, and T. Yamashita, "Implementation of new superconducting neural circuits using coupled squids," IEEE Transactions on Applied Superconductivity, vol. 4, pp. 1-8, May 1994.

[8] T. V. Filippov, Y. A. Polyakov, V. K. Semenov, and K. K. Likharev, "Signal resolution of RSFQ comparators," IEEE Transactions on Applied Superconductivity, vol. 5, pp. 2240-2243, June 1995.

[9] Y. Yamanashi, K. Umeda, and N. Yoshikawa, "Pseudo sigmoid function generator for a superconductive neural network," IEEE Trans. on Applied Superconductivity, vol. 23, pp. 1701004, June 2003.

[10] A. E. Schegolev, N. V. Klenov, I. I. Soloviev, and M. V. Tereshonok, "Adiabatic superconducting cells for ultra-low-power artificial neural networks," Beilstein J Nanotechnol, vol. 7, pp. 1397-1403, October 2016.

[11] Submitted to publication. 